\title{
Diffraction and the Pomeron
}

\author{
H. Abramowicz \\ School of Physics and Astronomy \\ Raymond and Beverly Sackler Faculty of Exact Sciences \\ Tel Aviv University, Israel
}

\section{Introduction}

In hadron-hadron scattering, interactions are classified by the characteristics of the final states. In elastic scattering, both hadrons emerge unscathed and no other particles are produced. In diffractive scattering, the energy transfer between the two interacting hadrons remains small, but one (single dissociation) or both (double dissociation) hadrons dissociate into multi-particle final states, preserving the quantum numbers of the associated initial hadron. The remaining configurations correspond to inelastic interactions.

The first interpretation of diffraction, due to Good and Walker [1], was that different components of the projectile were differently absorbed by the target, leading to the creation of new physical states. This was the first indication for the composite nature of hadrons.

In the Regge theory of strong interactions [2], diffraction is the result of exchanging a universal trajectory with the quantum numbers of the vacuum, the (soft) Pomeron, $\mathbb{P}$, introduced by Gribov [3].

In the language of Quantum Chromodynamics, the candidate for vacuum exchange with properties similar to the soft Pomeron is two gluon exchange [4, 5]. As a result of interactions between the two gluons, a ladder structure develops. In perturbative QCD, the properties of this ladder depend on the energy and scales involved in the interaction, implying its non-universal character. In the high-energy limit, the properties of the ladder have been derived for multi-Regge kinematics and the resulting exchange is called the (hard) BFKL pomeron [6, 7, 8].

A renewed interest in diffractive scattering followed the observation of a copious production of diffractive-like events in deep inelastic scattering (DIS) at the HERA ep collider [9, 10] as well as the earlier observation of jet production associated with a leading proton in $p \bar{p}$ at CERN [1]]. The presence of a large scale opens the possibility of studying the partonic structure of the diffractive exchange as suggested by Ingelman and Schlein [12] and testing QCD dynamics. Moreover, the study of diffractive scattering offers a unique opportunity to understand the relation between 
the fundamental degrees of freedom prevailing in soft interactions - hadrons and Regge trajectories, and those of QCD - quarks and gluons. One of the challenges is to establish theoretically and experimentally the reactions in which the soft component is dominant and those in which the perturbative QCD formalism is applicable. In this report the discussion will focus on single diffractive processes in the presence of a large scale, mainly studied at HERA and at FNAL.

\section{Kinematics of diffractive scattering}

The variables used to analyze diffractive scattering will be introduced for ep DIS. Since DIS is perceived as a two-step process, in which the incoming lepton emits a photon which then interacts with the proton target, the relevant variables can be readily generalized to $p \bar{p}$ interactions.

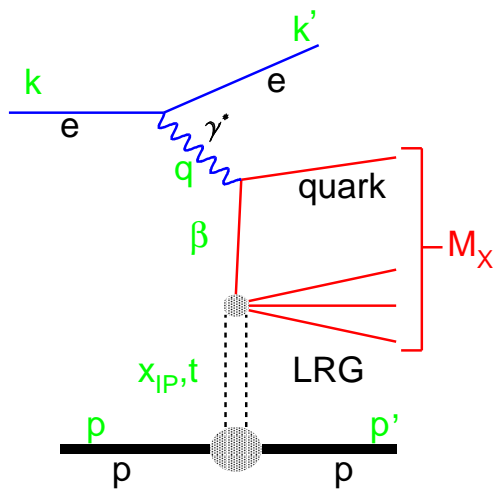

Figure 1: Schematic diagram for diffractive DIS in ep interactions.

A diagram for diffractive scattering in DIS, where the diffracted state is separated from the scattered proton by a large rapidity gap (LRG), is presented in Figure 1 and all the relevant four vectors are defined therein. In addition to the usual DIS variables, $Q^{2}=-q^{2}=-\left(k-k^{\prime}\right)^{2}, W^{2}=(q+p)^{2}, x=\frac{Q^{2}}{2 p \cdot q}$ and $y=\frac{p \cdot q}{p \cdot k}$, the variables used to described the diffractive final state are,

$$
\begin{aligned}
t & =\left(p-p^{\prime}\right)^{2}, \\
x_{\mathbb{P}} & =\frac{q \cdot\left(p-p^{\prime}\right)}{q \cdot p} \simeq \frac{M_{X}^{2}+Q^{2}}{W^{2}+Q^{2}}, \\
\beta & =\frac{Q^{2}}{2 q \cdot\left(P-P^{\prime}\right)}=\frac{x}{x_{\mathbb{P}}} \simeq \frac{Q^{2}}{Q^{2}+M_{X}^{2}} .
\end{aligned}
$$

$x_{\mathbb{P}}$ is the fractional loss of the proton longitudinal momentum. It is sometimes denoted by $\xi$. $\beta$ is the equivalent of Bjorken $x$ but relative to the exchanged object. 
$M_{X}$ is the invariant mass of the hadronic final state recoiling against the leading proton, $M_{X}^{2}=\left(q+p-p^{\prime}\right)^{2}$. The approximate relations hold for small values of the four-momentum transfer squared $t$ and large $W$, typical of high energy diffraction.

The need to preserve the identity of the target in diffractive scattering limits the square of the momentum transfer, $|t|<1 / R_{T}^{2}$, where $R_{T}$ is the radius of the target. The $t$ distribution typically has an exponential behaviour, $f(t) \sim \exp (-b|t|)$ with $b \simeq R_{T}^{2} / 6$. The allowed $M_{X}$ is also limited by the coherence requirement. The minimum value of $t$ required to produce a given $M_{X}$ from a target with mass $m_{T}$ is $\left|t_{\min }\right| \simeq m_{T}^{2}\left(M_{X}^{2}+Q^{2}\right)^{2} / W^{4}$. For a typical hadronic radius of $1 \mathrm{fm}, M_{X}^{2}<0.2 W^{2}$ and the hadronic final state exhibits a large rapidity gap between the fragments of the diffracted state and the unscathed target (see Figure 2). Therefore in collider

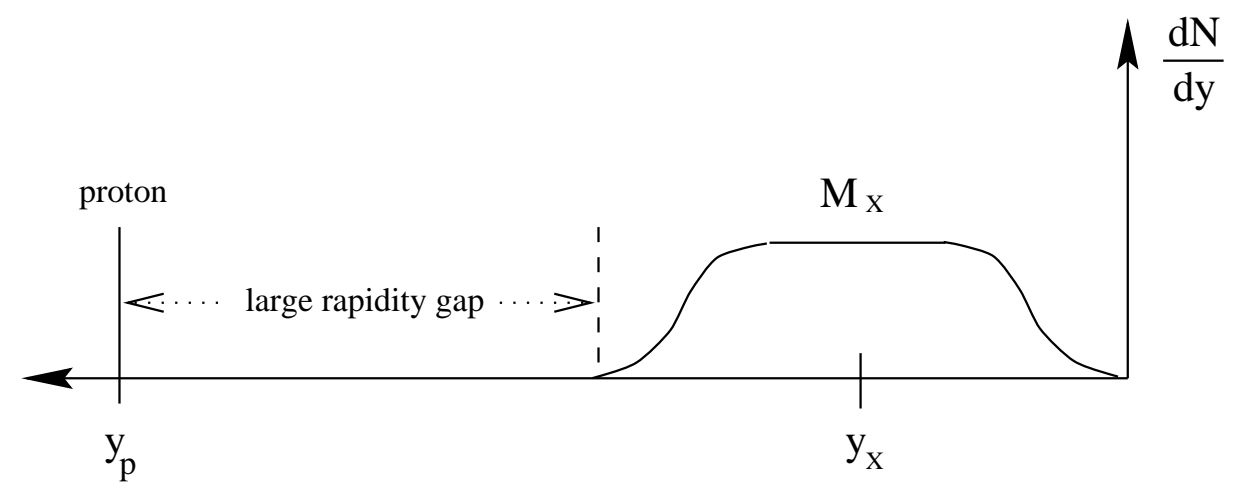

Figure 2: Schematic representation of rapidity $(y)$ distribution for single diffraction events

experiments, diffractive events are identified either by the presence of a fast proton along the beam direction or by the presence of a large rapidity gap in the central detectors.

\section{Formalism of diffractive scattering}

To describe diffractive DIS, it is customary to choose the variables $x_{\mathbb{P}}$ and $t$ in addition to the usual $x$ and $Q^{2}$ in the cross section formula. The four-fold differential cross section for $e p$ scattering can be written as

$$
\frac{d^{4} \sigma_{e p}^{D}}{d x_{\mathbb{P}} d t d x d Q^{2}}=\frac{2 \pi \alpha^{2}}{x Q^{4}}\left[1+(1-y)^{2}\right] F_{2}^{D(4)}\left(x, Q^{2}, x_{\mathbb{P}}, t\right),
$$

where for the sake of simplicity the contribution from the longitudinal structure function is omitted. The superscript $D$ denotes the diffractive process and the number 
in parenthesis in the superscript of the $F_{2}^{D}(4)$ is a reminder that the units of the structure function have changed. $F_{2}^{D(4)}$ integrated over $t$ is denoted by $F_{2}^{D(3)}$.

The structure function $F_{2}$ is related to the absorption cross section of a virtual photon by the proton, $\sigma_{\gamma^{\star} p}$. For diffractive scattering, in the limit of high $W$ (low $x$ ),

$$
F_{2}^{D(4)}\left(x, Q^{2}, x_{\mathbb{P}}, t\right)=\frac{Q^{2}}{4 \pi^{2} \alpha} \frac{d^{2} \sigma_{\gamma^{\star} p}^{D}}{d x_{\mathbb{P}} d t} .
$$

This relation allows predictions for diffractive scattering in DIS based on Regge phenomenology applied to $\gamma^{\star} p$ scattering. In fact many of the questions that are addressed in analyzing diffractive scattering are inspired by Regge phenomenology as established in soft hadron-hadron interactions.

\subsection{Regge phenomenology}

For scattering of two hadrons $a$ and $b$ at squared center of mass energy $s \gg m_{a, b}^{2}, t$, Regge phenomenology implies that,

$$
\begin{aligned}
\sigma_{t o t}(a b) & \sim s^{\alpha_{\mathbb{P}}(0)-1}, \\
\frac{d^{2} \sigma^{e l}}{d t}(a b \rightarrow a b) & =\frac{\sigma_{t o t}^{2}(a b)}{16 \pi} e^{2\left(b_{0}^{e l}+\alpha_{\mathbb{P}}^{\prime} \ln s\right) t}, \\
\frac{d^{2} \sigma^{D}}{d t d M_{X}^{2}}(a b \rightarrow X b) & \sim \frac{1}{M_{X}^{2}}\left(\frac{s}{M_{X}^{2}}\right)^{2\left(\alpha_{\mathbb{P}}(0)-1\right)} e^{2\left(b_{0}^{D}+\alpha_{\mathbb{P}}^{\prime} \ln \frac{s}{M_{X}^{2}}\right) t},
\end{aligned}
$$

where $\alpha_{\mathbb{P}}(t)=\alpha_{\mathbb{P}}(0)+\alpha_{\mathbb{P}}^{\prime} t$ is the parameterization of the $\mathbb{P}$ trajectory . The universality of this parameterization has been pointed out by Donnachie and Landshoff. The value of $\alpha_{\mathbb{P}}(0)=1.081$ [13] and $\alpha_{\mathbb{P}}^{\prime}=0.25 \mathrm{GeV}^{-2}$ [14] were derived based on total hadron-proton interaction cross sections and elastic proton-proton data. Recently the $\mathbb{P}$ intercept has been reevaluated [15] leading to a value of $\alpha_{\mathbb{P}}(0)=1.096 \pm 0.03$. The $\mathbb{P}$ intercept is sometimes presented as $\alpha_{\mathbb{P}}(0)=1+\epsilon$.

Three implications are worth noting.

(1) The slope of the $t$ distribution is increasing with $\ln s$. This fact, borne out by the data, is known as shrinkage of the $t$ distribution. It is due to the fact that $\alpha_{\mathbb{P}}^{\prime} \neq 0$ and has been explained by Gribov [3] as diffusion of particles in the exchange towards low transverse momenta, $k_{T}$, with $\alpha_{I P}^{\prime} \sim 1 / k_{T}^{2}$ (see also [16]).

(2) A steep and universal $x_{\mathbb{P}}$ dependence of the diffractive cross section is expected, $d \sigma^{D} / d x_{\mathbb{P}} \sim x_{\mathbb{P}}^{-(1+2 \epsilon)}$.

(3) The ratios $\sigma^{e l} / \sigma_{\text {tot }}$ and $\sigma^{D} / \sigma_{\text {tot }}$ rise like $s^{\epsilon}$. Since $\epsilon>0$ this is bound to lead to unitarity violation. 


\subsection{Perturbative QCD}

QCD factorization for the diffractive structure function of the proton, $F_{2}^{D}$, is expected to hold [17, 18, 19]. $F_{2}^{D}$ is decomposed into diffractive parton distributions , $f_{i}^{D}$, in a way similar to the inclusive $F_{2}$,

$$
\frac{d F_{2}^{D}\left(x, Q^{2}, x_{\mathbb{P}}, t\right)}{d x_{\mathbb{P}} d t}=\sum_{i} \int_{0}^{x_{\mathbb{P}}} d z \frac{d f_{i}^{D}\left(z, \mu, x_{\mathbb{P}}, t\right)}{d x_{\mathbb{P}} d t} \hat{F}_{2, i}\left(\frac{x}{z}, Q^{2}, \mu\right)
$$

where $\hat{F}_{2, i}$ is the universal structure function for DIS on parton $i, \mu$ is the factorization scale at which $f_{i}^{D}$ are probed and $z$ is the fraction of momentum of the proton carried by the diffractive parton $i$. Diffractive partons are to be understood as those which lead to a diffractive final state. The DGLAP evolution equation applies in the same way as for the inclusive case. For a fixed value of $x_{I P}$, the evolution in $x$ and $Q^{2}$ is equivalent to the evolution in $\beta$ and $Q^{2}$.

Note that QCD factorization for the diffractive parton distributions is expected to fail for hard diffraction in hadron collisions [17].

If, following Ingelman and Schlein [12], one further assumes the validity of Regge factorization, $F_{2}^{D}$ may be decomposed into a universal $\mathbb{P}$ flux and the structure function of $\mathbb{P}$,

$$
\frac{d F_{2}^{D}\left(x, Q^{2}, x_{\mathbb{P}}, t\right)}{d x_{\mathbb{P}} d t}=f_{\mathbb{P} / p}\left(x_{\mathbb{P}}, t\right) F_{2}^{\mathbb{P}}\left(\beta, Q^{2}\right)
$$

where the normalization of either of the two components is arbitrary. It implies that the $x_{\mathbb{P}}$ and $t$ dependence of the diffractive cross section is universal, independent of $Q^{2}$ and $\beta$, and given by (see formula 8)

$$
f_{\mathbb{P} / p}\left(x_{\mathbb{P}}, t\right) \sim\left(\frac{1}{x_{\mathbb{P}}}\right)^{2 \alpha_{\mathbb{P}}(0)-1} e^{2\left(b_{0}^{D}-\alpha_{\mathbb{P}}^{\prime} \ln x_{\mathbb{P}}\right) t} .
$$

In this approach, the diffractive parton distributions would be obtained as a convolution of the $\mathbb{P}$ flux with parton distributions in the $\mathbb{P}$.

None of the approaches detailed above address the issue of the dynamical origin of the $\mathbb{P}$ exchange in perturbative QCD. The mechanism for producing LRG is assumed to be present at some scale and the evolution formalism allows to probe the underlying partonic structure. A more dynamical approach will be discussed in the context of the measurements performed up to date.

\section{Measurements of $F_{2}^{D}$ at HERA}

The data analyzed to date come from $e^{+} p$ runs with the proton beam momentum of $820 \mathrm{GeV}$ and positron beam of $27.5 \mathrm{GeV}$. The coverage of phase space in $\beta$ and $Q^{2}$ as well as $\beta$ and $x_{\mathbb{P}}$ is shown in Figure 3 . 

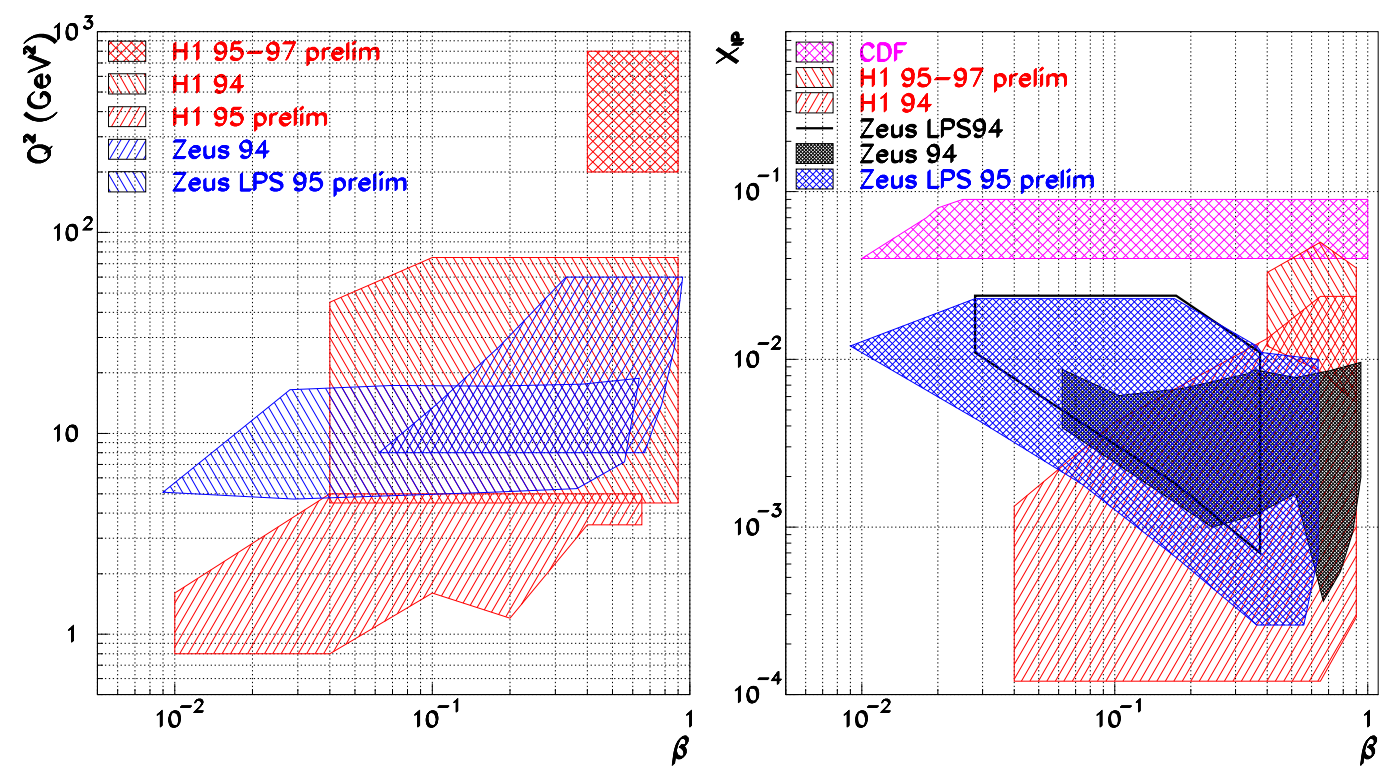

Figure 3: Phase space coverage in $\beta, Q^{2}$ and $x_{I P}$ for the measurements of $F_{2}^{D}$ in $e p$ interactions. Also shown is the $x_{\mathbb{P}}$ and $\beta$ coverage of the recent CDF data with the recoil proton measured in the Roman pots (see section 6.2).

\subsection{Energy dependence and Regge factorization}

Following the lead of Regge phenomenology, the energy dependence of $\sigma^{D}\left(\gamma^{\star} p\right)$ or the $x_{\mathbb{P}}$ dependence of $F_{2}^{D}$ in the data is expressed in terms of $\alpha_{\mathbb{P}}(0)$. For $x_{\mathbb{P}}<0.01$ and within a given range of $Q^{2}$ the data are compatible with a universal $x_{\mathbb{P}}$ dependence [20, 21, 22. The data used for these studies are integrated over $t$. To derive the value of $\alpha_{\mathbb{P}}(0)$, it is assumed that the slope of the $t$ distribution is $Q^{2}$ independent and is given by $b^{D}=7 \mathrm{GeV}^{-2}$ [23]. For lack of other information $\alpha_{I P}^{\prime}=0.25 \mathrm{GeV}^{-2}$ is used. The $Q^{2}$ dependence of $\alpha_{\mathbb{P}}(0)$ derived from diffractive measurements is shown in Figure 4 . For comparison, also shown is the $Q^{2}$ dependence of $\alpha_{\mathbb{P}}(0)$ derived from the inclusive DIS measurements and conveniently represented by the ALLM97 parameterization [24]. As has been known for a while, the inclusive DIS data at high $W$ are not compatible with a universal $\mathbb{P}$ trajectory. They are however in very good agreement with expectations of QCD evolution. The diffractive measurements seem to point to some $Q^{2}$ dependence, but less pronounced than that in the inclusive case. For $Q^{2}>10 \mathrm{GeV}^{2}$ the value of $\alpha_{\mathbb{P}}(0)$ is significantly higher than measured for soft interactions. The important observation is that the $W$ dependence of diffractive DIS is the same as for the inclusive cross section [21] and slower than would be expected from Regge phenomenology. 


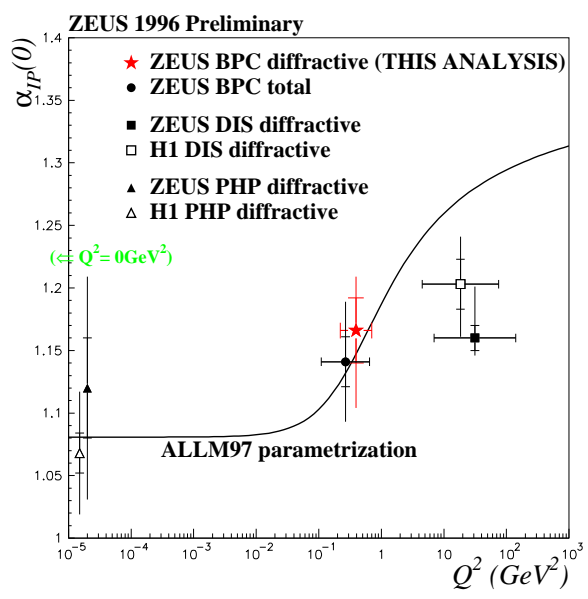

Figure 4: $Q^{2}$ dependence of $\alpha_{\mathbb{P}}(0)$ derived from measurements of diffractive and total $\gamma^{\star} p$ cross sections. The curve (ALLM97) is a representation of the results obtained in inclusive DIS measurements.

\section{2 $Q^{2}$ and $\beta$ dependence}

The $Q^{2}$ dependence of $x_{\mathbb{P}} F_{2}^{D(3)}$ as measured by the H1 experiment 20, 25] for two values of $x_{\mathbb{P}}$ and for a range of $\beta$ values is shown in Figure 5. Also shown in the figure is the $\beta$ distribution measured with the leading proton spectrometer (LPS) of the ZEUS detector, at $x_{\mathbb{P}}=0.01$ for two values of $Q^{2}$ [26]. Both the $Q^{2}$ and the $\beta$ dependence of $F_{2}^{D}$ are very different from the one known for the proton $F_{2}$. There is almost no $Q^{2}$ dependence for $\beta>0.4$ and the $\beta$ distribution itself is relatively flat up to large $\beta \simeq 1$. This suggests a very different parton content of diffractive scattering compared to the inclusive scattering. As we will see below, for the DGLAP evolution to hold, a large gluon component is required at large $\beta$ to compensate for the loss of quark momenta in the evolution.

\subsection{Diffractive parton distributions}

The diffractive parton density functions (DPDF) are derived from $F_{2}^{D}$ by fitting the DGLAP evolution equations with postulated input $\beta$ distributions at some low $Q_{0}^{2}$ scale. The fit consists of adjusting the parameters of the input distributions to get the best description of all available data. No momentum sum-rule is available for diffractive partons, however one assumes flavor symmetry for light quarks. The results of such a fitting procedure [20] are shown in Figure 6. Different solutions are found, however for all cases a large gluon component is required. The net result is that while the density of inclusive quark distributions (represented by the flavor singlet structure function $\Sigma$ ) decreases with $Q^{2}$ at $x=0.2$, the density of diffractive partons 

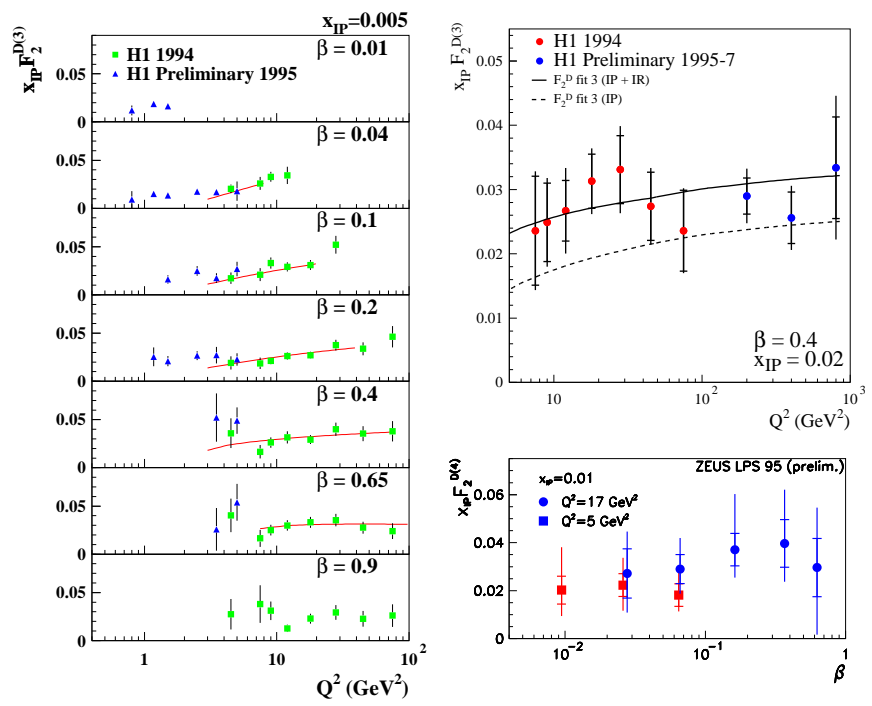

Figure 5: Left and upper right plots: $Q^{2}$ dependence of $x_{\mathbb{P}} F_{2}^{D(3)}$ at fixed values of $\beta$ and $x_{\mathbb{P}}$ as denoted in the figure. Lower right plot: $\beta$ dependence of $x_{\mathbb{P}} F_{2}^{D(4)}$ (for $0.073<|t|<0.4 \mathrm{GeV}^{2}$ ) at fixed values of $Q^{2}$ and $x_{\mathbb{P}}$ as denoted in the figure.

is still rising with $Q^{2}$ at the equivalent $\beta=0.2$ [27].

\subsection{Jet and charm production}

The partonic structure of diffractive scattering can be further explored by studying the hadronic final states. A large gluon component is expected to lead to a copious production of high $p_{T}$ jets as well as of charm, through the boson-gluon fusion diagram depicted in Figure 77. The H1 experiment has measured the cross section for dijet production in diffractive scattering [28]. The observed rates are in agreement with expectations based on DPDFs obtained from fits to $F_{2}^{D}$.

Diffractive charm production has also been measured [29, 30], however while ZEUS measurements are in agreement with expectations, the $\mathrm{H} 1$ result seems to be smaller than expected. Note that the statistical and systematic errors of these measurements are quite large and a definite answer awaits more data.

\section{$5 \quad$ Generic model for diffractive DIS}

A tremendous theoretical effort has been, and still is, devoted to the understanding of the dynamics behind diffractive DIS (for a recent review see [31, 32] and references therein(]). A simple picture of diffraction emerges if the process is viewed in the rest

${ }^{1}$ List of representative papers from which a thorough exploration can begin: 33, 34, 35, 36, 37, 38, 39, 40. 

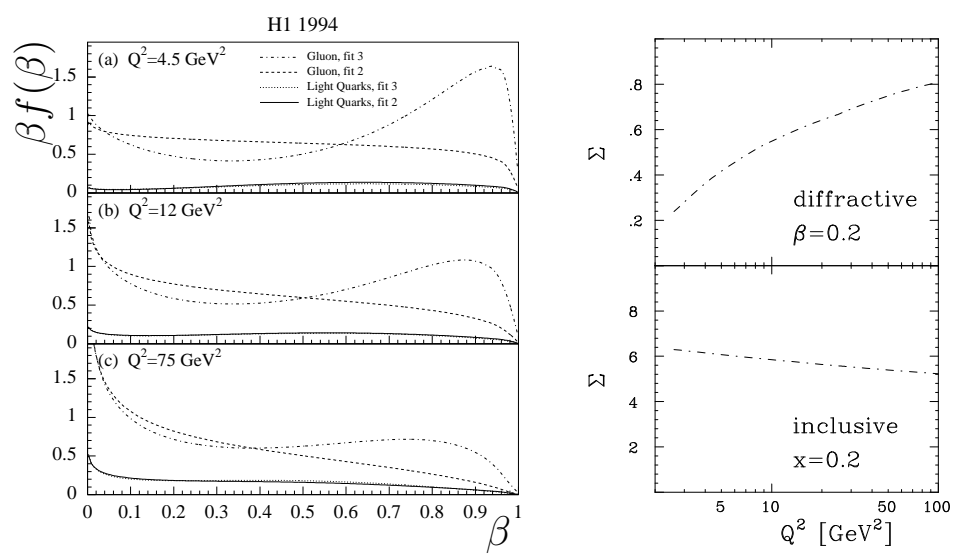

Figure 6: Left: diffractive parton densities $\beta f(\beta)$ as a function of $\beta$ obtained by fitting the DGLAP evolution equations. Right: difference in $Q^{2}$ evolution of the singlet structure function $\Sigma$ for diffractive and inclusive parton distributions for $\beta=0.2$ and $x=0.2$ respectively.
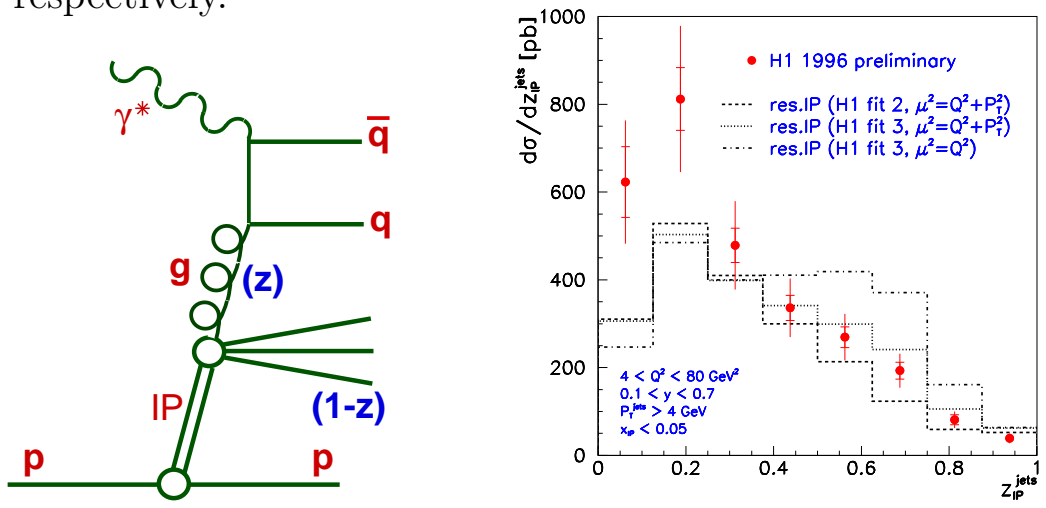

Figure 7: Left: boson-gluon fusion diagram. Right: The dependence of diffractive dijet cross section on $z_{\mathbb{P}}^{\text {jets }}$, the experimental estimate of $z$ as defined in the left diagram. The curves correspond to expectations for different DPDFs.

frame of the proton. The virtual photon develops a partonic fluctuations, whose lifetime is $\tau=1 / 2 m_{p} x$ [41]. At the small $x$ typical of HERA, where $\tau \sim 10-100 \mathrm{fm}$, it is the partonic state rather than the photon that scatters off the proton. If the scattering is elastic, the final state will have the features of diffraction.

The equivalence between the approach in the proton rest frame and in the Breit frame is schematically depicted in Figure 8 [42]. An interaction initiated by fluctuation of $\gamma^{\star}$ into a $q \bar{q}(q \bar{q} g)$ state will contribute to the diffractive quark (gluon) distribution. The fluctuations of the $\gamma^{\star}$ are described by the wave functions of the transversely and longitudinally polarized $\gamma^{\star}$ which are known from perturbative QCD. Small and large partonic configurations of the photon fluctuation are present. For large configurations non-perturbative effects dominate in the interaction and the treatment of 

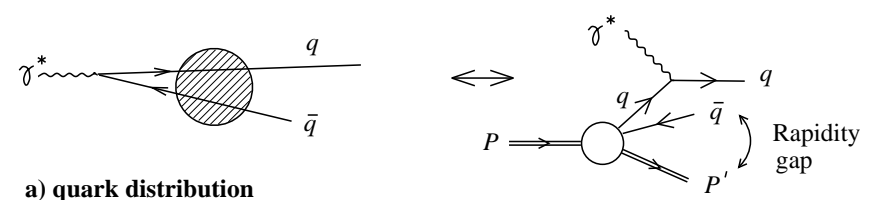

a) quark distribution

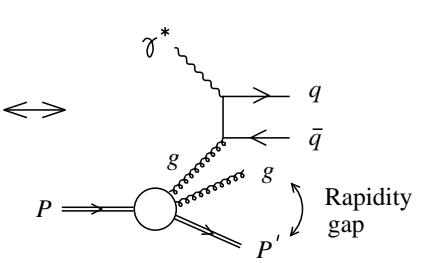

b) gluon distribution

Figure 8: Schematic representation of the equivalence between diffractive DIS in the proton rest frame (left) and in the Breit frame (right).

this contribution is subject to modeling. For a small configuration of partons (large relative $k_{T}$ ) the total interaction cross section of the created color dipole on a proton target is given by 43, 44

$$
\begin{aligned}
\sigma_{q \bar{q} p} & =\frac{\pi^{2}}{3} r^{2} \alpha_{S}(\mu) x g(x, \mu) \\
\sigma_{q \bar{q} g p} & \simeq \sigma_{g g p}=\frac{9}{4} \sigma_{q \bar{q} p}
\end{aligned}
$$

where $r$ is the transverse size of the color dipole and $\mu \sim 1 / r^{2}$ is the scale at which the gluon distribution $g$ of the proton is probed. The corresponding elastic cross section is obtained from the optical theorem. In this picture, the gluon dominance in diffraction results from the dynamics of perturbative QCD (see equation (13)).

The interesting observation is that all the models, whether starting from a purely perturbative approach [27] or a semi-classical approach [35], give a good description of the $Q^{2}$ and $\beta$ dependence of $F_{2}^{D}$. The leading-twist behavior of diffractive DIS is determined by the hadronic nature of the $\gamma^{\star} p$ interactions. The $\beta$ dependence seems to be mostly determined by the wave function of $\gamma^{\star}$.

A possible explanation of the success of the perturbative approach to diffractive DIS has been proposed by Mueller [45. If the large size $\gamma^{\star}$ fluctuations were to be absorbed, diffraction would be dominated by small size configurations which lend themselves to perturbative calculations. The absorption would be a manifestation of unitarity effects and would explain the slower than expected $W$ dependence of the $\gamma^{\star} p$ diffractive cross section (see section 4.1). Absorption effects have been successfully incorporated in some of the models 36, 37.

The possibility that unitarity effects at low $x$ are not negligible has been pointed out by Frankfurt and Strikman [44]. The probability that an interaction on parton $i$ 
is associated with a diffractive final state, $P_{i}^{D}$, is defined as

$$
P_{i}^{D}\left(x, Q^{2}\right)=\frac{\int_{t, x_{\mathbb{P}}} f_{i}^{D}\left(\frac{x}{x_{\mathbb{P}}}, Q^{2}, x_{\mathbb{P}}, t\right) d x_{\mathbb{P}} d t}{f_{i}\left(x, Q^{2}\right)},
$$

where $f_{i}$ stands for the inclusive density of parton $i . P_{i}^{D}$ may be interpreted as the ratio of elastic to total cross sections, $r_{e l}(i)=\sigma_{e l}(i) / \sigma_{t o t}(i)$, induced either by a $q \bar{q}$ $(i=q)$ or $q \bar{q} g(i=g)$ configurations of $\gamma^{\star}$. In the black disc limit, $r_{e l}=0.5$. In DIS, for $Q^{2}=4.5 \mathrm{GeV}^{2}$ and $x<10^{-3}$, the calculation using the ACTW 46] parameterization leads to $P_{g}^{D} \simeq 0.4$ for gluons and $P_{q}^{D} \simeq 0.15$ for quarks. The value of $P_{g}^{D}$, close to the black disc limit, may indicate the presence of unitarity effects in the gluon sector of the evolution.

\section{$6 \quad$ Diffraction in $p \bar{p}$ scattering}

Diffractive-like events have been observed in hard $p \bar{p}$ scattering by both the CDF and the D0 experiments. The signature of these events, depicted in Figure 9, is the presence of a large rapidity gap in one of the forward regions associated with the presence of either large transverse momentum jets [47, 48], beauty [49], $J / \psi$ [50] or the $W^{ \pm}$boson [51].

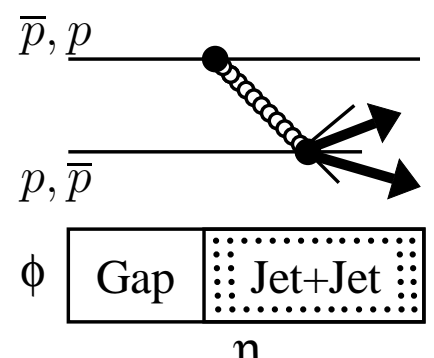

$\eta$

Figure 9: Schematic representation of single diffractive events in $p \bar{p}$ collisions and the corresponding density of the phase space of the azimuthal angle $\phi$ and of pseudorapidity $\eta$.

\subsection{Rates of diffractive processes}

A compilation of the published and preliminary results on the ratio of diffractive to inclusive events with a given hadronic final state is presented in Figure 10 as a function of the typical average scale of the process. The measurements are compared 
to expectations obtained using the ACTW-fit D parameterization [46] of DPDFs. In order to reproduce vaguely the measured numbers, the expectations have to be scaled down by a factor 20. This may not be surprising as QCD factorization is not applicable to hard diffractive scattering in hadron collisions [17]. What might be surprising is that the overall scale dependence is quite well reproduced. Unfortunately there are no calculations available for $p \bar{p}$ collisions at $\sqrt{s}=630 \mathrm{GeV}$. The difference in the contribution of the diffractive quarks and gluons to different processes has been used by CDF to constrain, to leading order, the momentum fraction $f_{g}$ of gluons relative to all diffractive partons. Including $W$, jet and beauty production, $f_{g}=0.54_{-0.14}^{+0.16}$, marginally less than in diffractive DIS, where this ratio is close to $90 \%$, for next-toleading order partons.

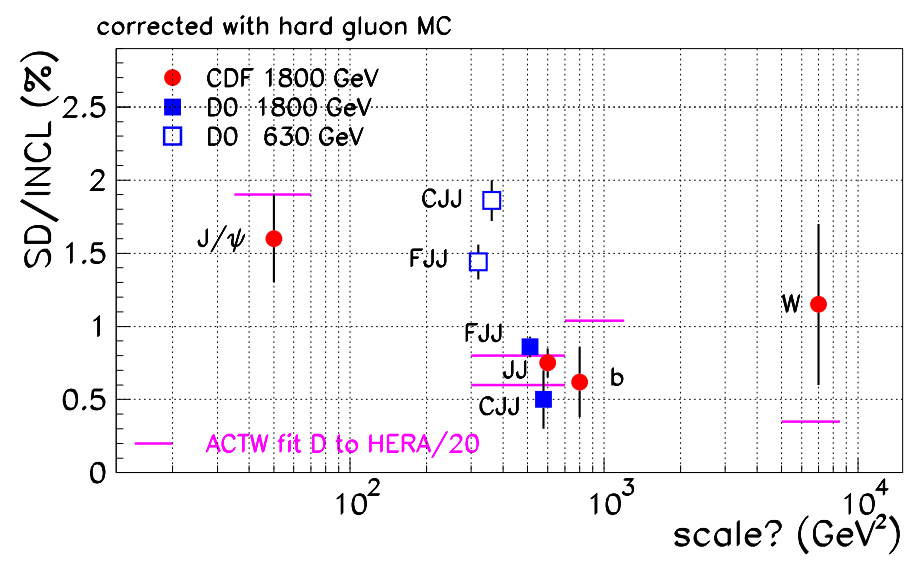

Figure 10: Ratio of single diffraction to inclusive production in $p \bar{p}, \mathrm{SD} / \mathrm{INCL}$, as a function of the approximate hard scale in the process. JJ stands for two jet production while $\mathrm{C}$ and $\mathrm{F}$ stand for forward and central production. Horizontal bars are expectations obtained using the ACTW parameterization, fit D 46], scaled down by factor 20 .

\section{$6.2 \xi$ and $\beta$ dependence}

The fractional momentum loss $\xi$ of the proton in hard diffractive jet production has been measured by D0 48. For $\sqrt{s}=1800$ (630) GeV, the distribution peaks at $\xi \simeq 0.03(0.07)$ and extends to $\xi=0.1(0.2)$. This is a region where contributions from sub-leading trajectories may become important.

CDF has measured $\xi$ and $\beta$ by detecting the leading $\bar{p}$ in a forward spectrometer consisting of Roman pots [52]. The results are expressed in terms of an effective structure function $F_{J J}^{D}$ derived from the rate of leading $\bar{p}$ and the two-jet inclusive cross section. The shape of the $\beta$ distribution was found to be independent of $\xi$, suggesting Regge factorization. For the measured range, $0.04<\xi<0.09$, the $\xi$ dependence of 
$F_{J J}^{D}$ at $\beta=0.2$ is described by $\xi^{-0.87}$. The $\beta$ distribution of $F_{J J}^{D}$ is shown in Figure 11 and compared to expectations using the H1 - fit 2 parameterization [20]. To get the right order of magnitude for $\beta>0.4$, the expectations have to be scaled down by a factor 20. In addition, CDF observes a relatively higher yield of low $\beta$ events. Without the power of the QCD factorization theorem and given the very different $\xi$

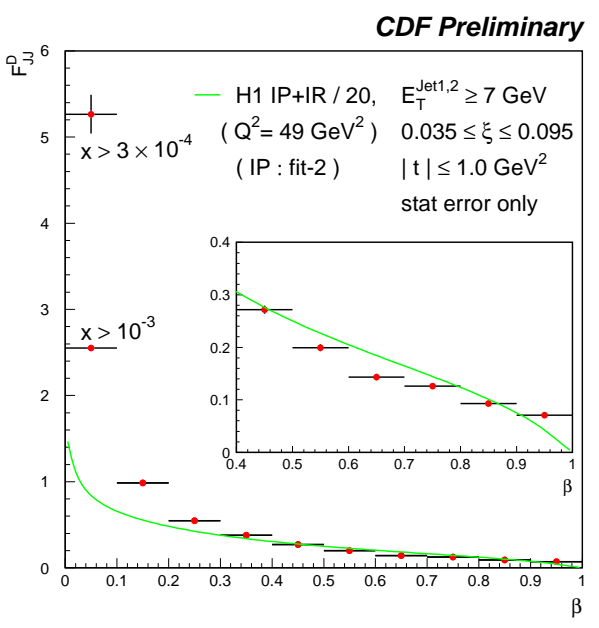

Figure 11: $\beta$ distribution of two jet production with a leading $\bar{p}$ in $p \bar{p}$ collisions compared to expectations based on diffractive parton distributions obtained at HERA [20] scaled down by factor 20 .

range, it is very difficult to draw any conclusions from the difference in the $p \bar{p}$ and $e p$ data.

\section{Vector meson production}

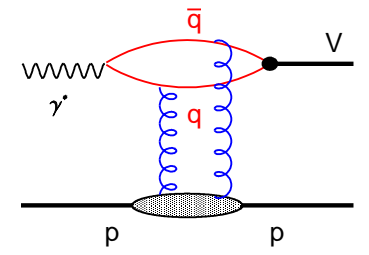

Figure 12: Schematic diagram for exclusive vector meson, $V$, production in hard diffractive scattering.

To test ideas behind the generic model of diffractive DIS (see section 5) and its hard component, one needs to devise a trigger to isolate when the photon fluctuates into a small-size $q \bar{q}$ configuration. A small-size $q \bar{q}$ dipole is most likely to be produced 
if the virtual photon is longitudinally polarized or if the dipole consists of heavy quarks such as $c \bar{c}$ or $b \bar{b}$. The exclusive $J / \psi$ [53] and $\Upsilon$ photoproduction as well as vector meson production in DIS [54] are likely candidates for hard diffractive processes fully calculable in perturbative QCD. The corresponding diagram is shown in Figure 12 . The predictions of perturbative QCD are very distinct:

(1) The flavor dependence of $\sigma_{V}=\sigma\left(\gamma^{\star} p \rightarrow V p\right)$ is determined by the photon quark current and the $\mathrm{SU}(4)$ relation

$$
\sigma_{\rho}: \sigma_{\omega}: \sigma_{\Phi}: \sigma_{J / \psi}=9: 1: 2: 8
$$

which is badly broken in soft interactions (see eg. [55]), is restored.

(2) Exclusive $V$ production in DIS is dominated by the longitudinal $\gamma_{L}^{*}$ component and [54

$$
\frac{d \sigma_{V, L}}{d t}=\frac{A}{Q^{6}} \alpha_{S}\left(Q^{2}\right)\left|x g\left(x, Q^{2}\right)\right|^{2},
$$

where $A$ depends on the $V$ wave function. At low $x$, the gluon distribution increases rapidly when $x$ decreases ( $W$ increases) and therefore a rapid increase of $\sigma_{V}$ with $W$ is expected.

(3) Because of the fast increase with $Q^{2}$ of gluons at low $x$, the expected $Q^{2}$ dependence is slower than $Q^{-6}$.

(4) The $t$ distribution should be universal, determined by the two-gluon form-factor, with $\alpha_{\mathbb{P}}^{\prime} \simeq 1 / Q^{2}$, because of the perturbative nature of the interaction.

Below some of the recent experimental findings are reviewed. A detailed discussion of HERA results can be found in [31].

\subsection{Flavour dependence}

A compilation of the ratio of $\Phi, \omega$ and $J / \psi$ production cross sections to that of the $\rho^{0}$ as a function of $Q^{2}$ is shown in Figure 13. The $\Phi: \rho^{0}$ and $J / \psi: \rho^{0}$ ratios increase
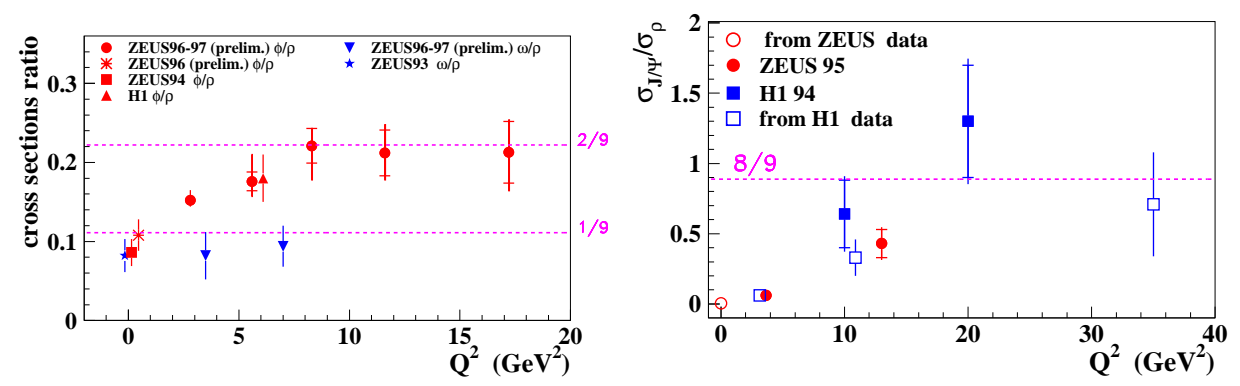

Figure 13: Cross sections ratio $\sigma_{V_{1}} / \sigma_{\rho^{0}}$ for $V_{1}=\Phi, \omega$ (left) and $J / \psi$ (right) as a function of $Q^{2}$.

with $Q^{2}$ and at high $Q^{2}$ reach, within errors, the value expected from $\mathrm{SU}(4)$. The $\omega: \rho^{0}$ remains constant. 


\section{2 $W$ dependence}

The $W$ dependence of exclusive $V$ photoproduction is shown in Figure 14. The striking fact is the fast rise of the $J / \psi$ cross section, which has now been measured by the $\mathrm{H} 1$ experiment up to $W=300 \mathrm{GeV}$ [56]. This rise is in good agreement with perturbative QCD calculations [58, 57]. For $\rho^{0}$ production [60, 59], the rise of the cross section with $W$ depends on $Q^{2}$, as shown in Figure 14. As can be seen, only at about $Q^{2}=20 \mathrm{GeV}^{2}$ the steepness of the rise is comparable to that of the $J / \psi$. This
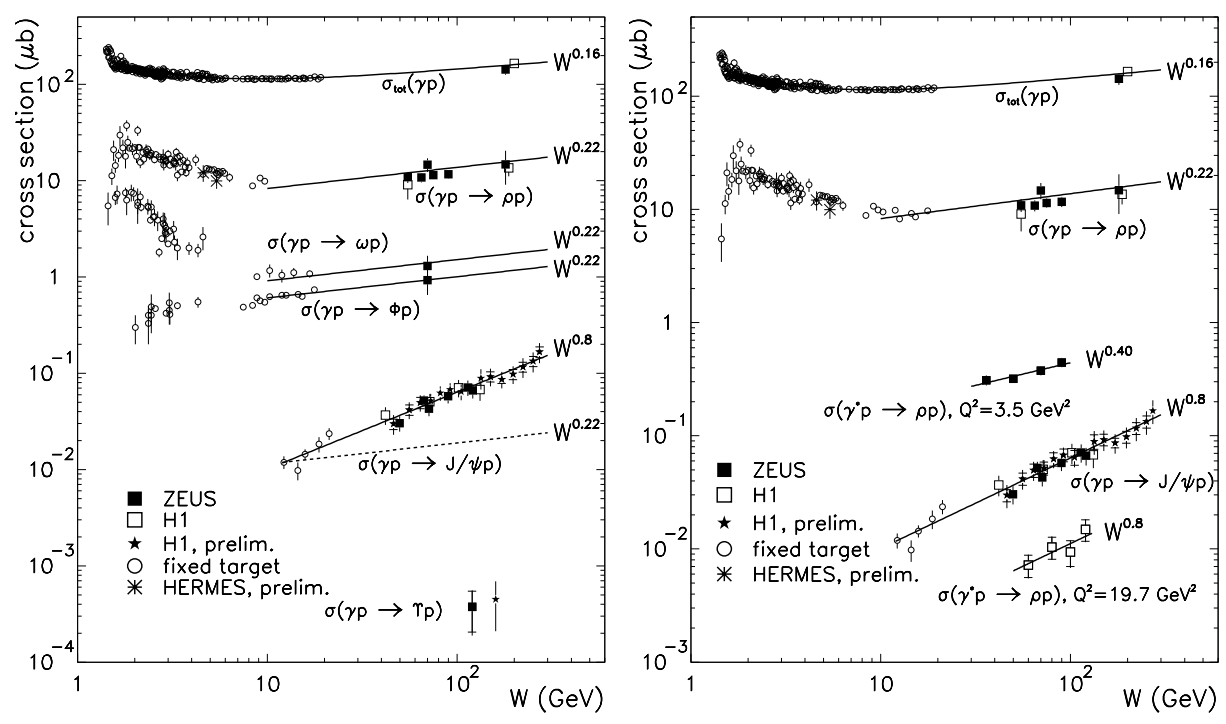

Figure 14: Left: $W$ dependence of the cross section $\sigma(\gamma p \rightarrow V p)$ for various vector mesons, from fixed target and HERA measurements. Right: $W$ dependence of $\rho^{0}$ cross section for different $Q^{2}$ values compared to $J / \psi$ photoproduction.

may be attributed to the effective scale in $V$ production, $Q_{e f f}^{2}$, which depends not only on $Q^{2}$ but also on wave function effects [58]. The rise with $W$ of $\sigma_{V} \sim W^{\delta}$ can be quantified by $\delta$, which is shown in Figure 15 as a function of $Q_{e f f}^{2}$. The results for different processes seem to line up along the same curve, which, above $Q_{e f f}^{2} \simeq 4 \mathrm{GeV}^{2}$, follows the expectations from the $W$ dependence of $\gamma^{\star} p$ total cross section.

Contrary to expectations, the $\Upsilon$ production cross section was found to be larger than predicted by perturbative calculations [61, 62]. This disagreement led to two findings [63, 64]. The gluons exchanged in $V$ production do not have the same value of $x$. For a $V$ with mass $M_{V}$ produced from a $\gamma^{\star}$ with virtuality $Q^{2}$, the difference $\delta x$ is given by

$$
\delta x=\frac{M_{V}^{2}+Q^{2}}{W^{2}+Q^{2}} .
$$

For $\Upsilon$ production, $\delta x$ can be large and the skewed gluon distribution has to be used. The skewed (also called off-forward) parton distributions are hybrid objects, which 


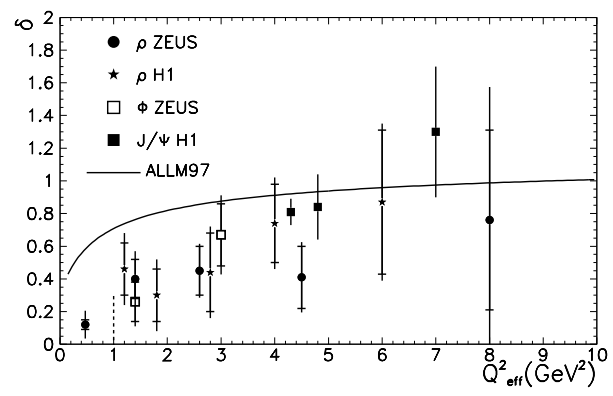

Figure 15: The power $\delta$ of the $W^{\delta}$ dependence of $\sigma\left(\gamma^{(\star)} p \rightarrow V p\right)$ as a function of the effective scale of the scattering, $Q_{\text {eff }}^{2}$. The curve represents the expectations from the $W$ dependence of the total $\gamma^{\star} p$ cross sections.

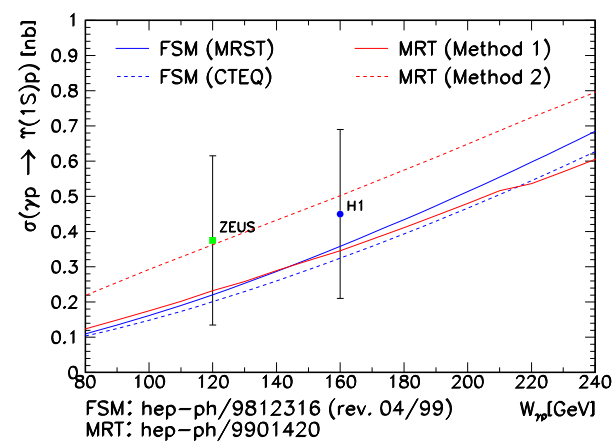

Figure 16: The $\Upsilon$ photoproduction cross section as a function of $W$ compared to calculations of perturbative QCD, FSM 63, MRT 64.

combine properties of form factors and ordinary parton distributions [65, 66]. The use of the skewed distribution leads to an enhancement of $\sigma_{\Upsilon}$ by a factor $\simeq 2$. For a process with a fast rise with $W$, the relation between the elastic cross section and the total cross section has the form

$$
\left.\frac{d \sigma_{e l}}{d t}\right|_{t=0}=\left(1+\left(\frac{\operatorname{Re} A}{\operatorname{ImA}}\right)^{2}\right) \frac{\sigma_{t o t}^{2}}{16 \pi},
$$

where the ratio of the real to imaginary part of the scattering amplitude, $A$, cannot be neglected. This produces another enhancement factor of $1.5-1.7$. The present agreement of theoretical calculations with data is shown in Figure 16.

\section{3 $Q^{2}$ dependence}

The $Q^{2}$ dependence of the cross section at fixed $W$ (ranging from 75 to $90 \mathrm{GeV}$ ) is parameterized as $\sigma_{V} \sim\left(Q^{2}+M_{V}^{2}\right)^{-n}$ 60, 59, 67, 68]. The power $n$ as a function 


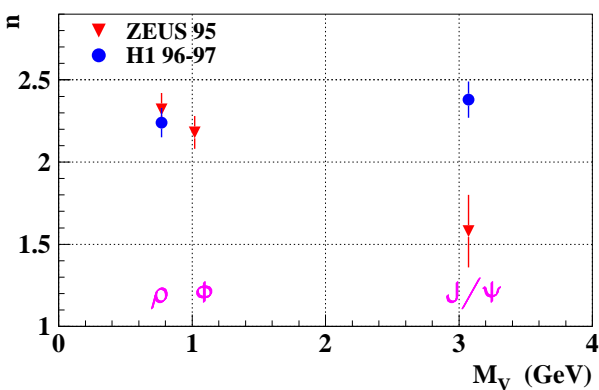

Figure 17: The power $n$ in the $\left(Q^{2}+M_{V}^{2}\right)^{-n}$ dependence of $V$ production as a function of mass, $M_{V}$.

of $M_{V}$ is shown in Figure 17 and is found to be substantially smaller than 3 (see equation (16)).

\section{$7.4 \quad t$ dependence}
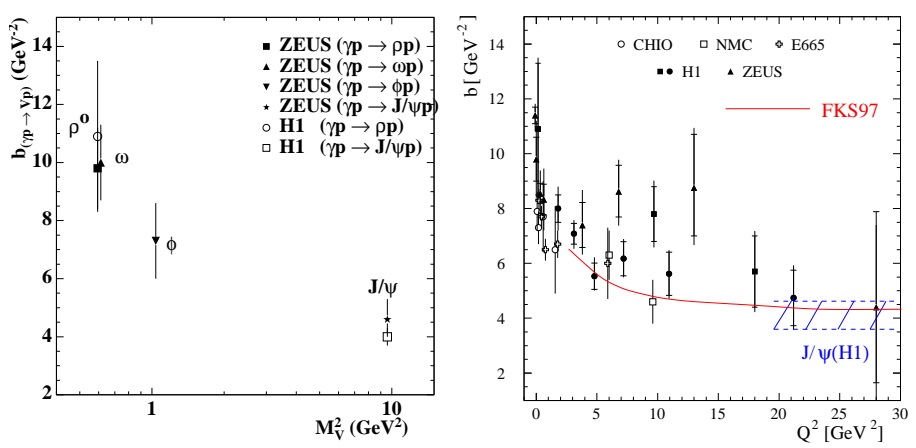

Figure 18: Left: the exponential slope $b$ of the $t$ distribution in $\gamma^{\star} p \rightarrow V p$ at HERA as a function of $M_{V}^{2}$. Right: the exponential slope $b$ as a function of $Q^{2}$ for $\rho^{0}$ production.

A compilation of the exponential slopes $b$ of the $t$ distributions at $Q^{2} \simeq 0$ as a function of $M_{V}$ is shown in Figure 18. For $\rho^{0}$ production, $b$ is also shown as a function of $Q^{2}$. The value of the slope decreases both with the mass of the $V$ and with $Q^{2}$, towards the value measured for the $J / \psi$, pointing to a decreasing size of the interaction region. The perturbative character of $J / \psi$ production can be best established by deriving the value of $\alpha_{P}^{\prime}$ from the $W$ dependence of the cross section at fixed $t$ (see equation (7))

$$
\frac{d \sigma}{d t} \sim W^{4\left(\alpha_{\mathbb{P}}(t)-1\right)} .
$$

Earlier measurements of HERA, combined with low energy data, indicated that $\alpha(t) \simeq$ const [69] (see Figure 19 left). H1 determined $\alpha_{\mathbb{P}}^{\prime}$ using only their own 
data [56]. The result, shown in Figure 19 with $\alpha_{I P}^{\prime}=0.05 \pm 0.15 \mathrm{GeV}^{-2}$, supports the hard nature of exclusive $J / \psi$ production at HERA.
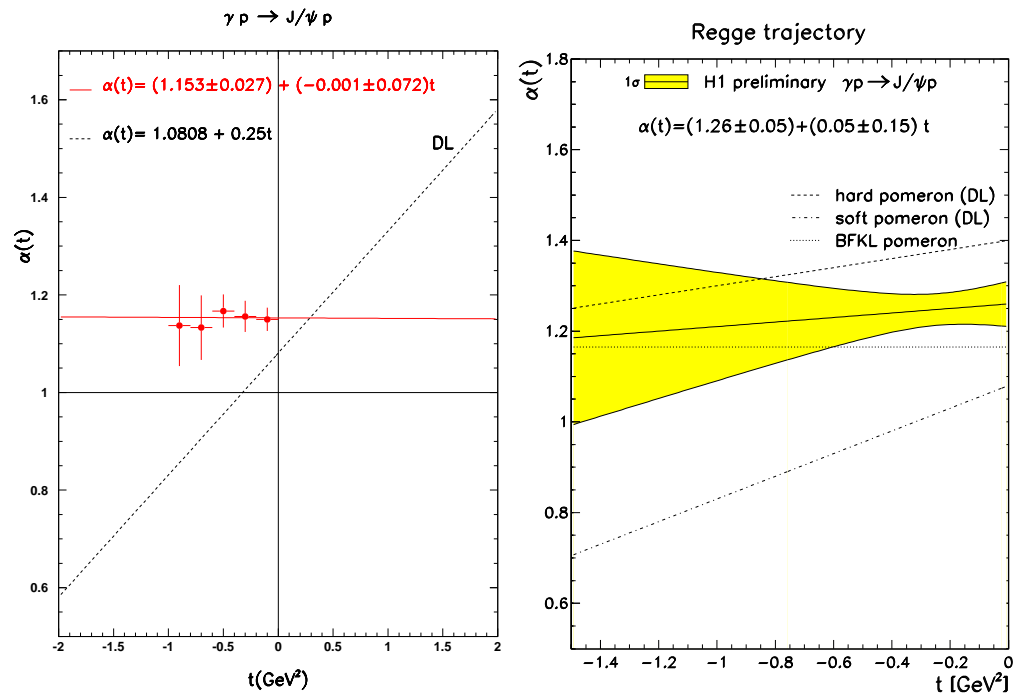

Figure 19: The Pomeron trajectory $\alpha_{\mathbb{P}}(t)$ as determined from low energy and HERA data (left) and from H1 data alone (right).

\section{Deeply Virtual Compton scattering}
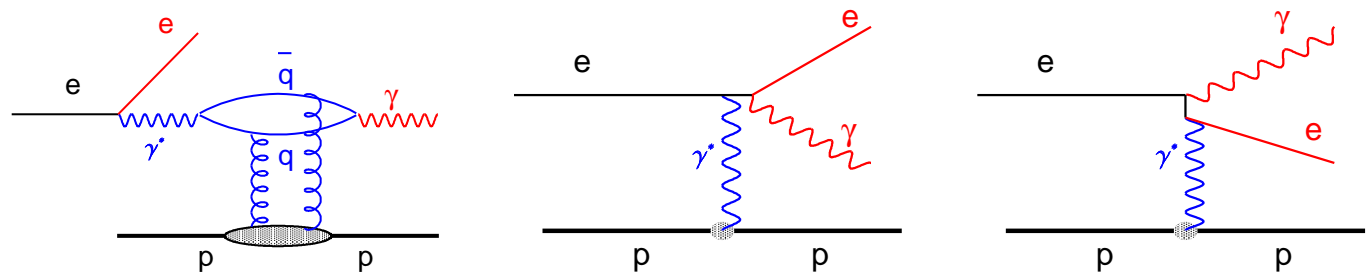

Figure 20: Diagrams for real $\gamma$ production in DIS.

Another example of hard diffractive scattering is the exclusive production of a real photon in DIS [70] (see Figure 20). In this deeply virtual Compton scattering (DVCS) (for recent discussions see 65, 66]), the cross section is less suppressed by factor $Q^{2}$ than in exclusive vector meson production [70], and the interpretation of the data is easier due to the lack of $V$ wave function effects. DVCS interferes with the QED Compton process, which leads to an identical final state, as depicted in Figure 20. The interference may allow a direct measurement of the real part of the 
QCD scattering amplitude. By extracting the DVCS, skewed parton distributions can be measured and additional information on the $F_{2}$ of the proton at low $x$ can be obtained 70$]$.

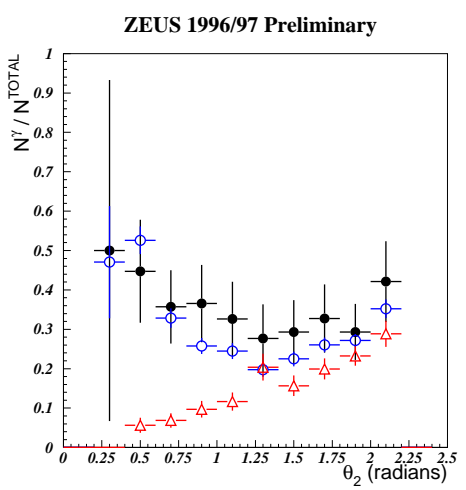

Figure 21: Fraction of photon candidates, $N^{\gamma} / N^{\mathrm{T} O T A L}$, for the second electromagnetic candidate in the calorimeter with energy $E>5 \mathrm{GeV}$, produced at angle $\theta_{2}$, closest to the proton direction. The data (dots) are compared to MC expectations for GenDVCS (circles) and for elastic Compton scattering only (triangles).

The ZEUS experiment has searched for DVCS in DIS events with $Q^{2}>6 \mathrm{GeV}^{2}$ and for $5 \cdot 10^{-4}<x<10^{-2}$ [71. Events with two electromagnetic, $e / \gamma$, candidates were selected with no additional hadronic activity in the central detector. The second candidate was defined as the one produced closest to the original proton direction, with angle $\theta_{2}$, and was required to be in the region of the detector where tracking is available. The data were compared to the expectations of a QED Compton MC [72] and a MC which included DVCS (GenDVCS) [73. The results are shown in Figure 21. A clear excess of $\gamma$ candidates at low $\theta_{2}$ is observed over expectations from the QED Compton process, while their rate is well reproduced by GenDVCS. The signal for DVCS is now established at HERA.

\section{Outlook}

This report is far from a complete account of diffractive physics. Its main purpose was to review the progress achieved in understanding diffractive phenomena with the language of perturbative QCD. Much has been learned from studying inclusive diffraction, where the gluons are found to be the main players. Diffractive DIS provides a complementary area to inclusive DIS in studying the dynamics of QCD at high energy. The new class of hard diffractive processes, such as exclusive vector meson production and deeply virtual Compton scattering, fulfill the properties expected from perturbative QCD. A comprehensive study of these processes may provide, in 
the future, important information on the gluon content in the proton and on the wave functions of vector mesons. The accumulated data cannot be described coherently by one universal Pomeron trajectory. The issue of what the Pomeron is requires further study.

Two subjects of potential interest, which were not discussed due to time constraints, are the large $t$ diffractive scattering studied at FNAL [74, 75] and HERA [76] and the double- $\mathbb{P}$ exchange studied at FNAL [77]. Here more theoretical work is necessary. It is quite clear that the emerging picture of diffraction will not be satisfactory without a complete account of all hard diffractive processes.

I would like to thank all my colleagues who have helped me in preparing this report. My special gratitude goes to M. Albrow, J. Bartels, K. Borras, A. Brandt, J. Dainton, K. Goulianos, L. Frankfurt, H. Kowalski, A. Levy, M. McDermott, N. Makins, K. Mauritz and M. Strikman who patiently discussed the data and their interpretation with me.

\section{References}

[1] M. L. Good and W. D. Walker, Phys. Rev. 120, 1857 (1960).

[2] P. D. Collins, "An Introduction To Regge Theory And High-Energy Physics," Cambridge University Press, $197 \%$.

[3] V. N. Gribov, JETP Lett. 41, 667 (1961).

[4] F. E. Low, Phys. Rev. D12, 163 (1975).

[5] S. Nussinov, Phys. Rev. Lett. 34, 1286 (1975).

[6] L. N. Lipatov, Sov. J. Nucl. Phys. 23, 338 (1976).

[7] E. A. Kuraev, L. N. Lipatov and V. S. Fadin, Sov. Phys. JETP 45, 199 (1977).

[8] I. I. Balitsky and L. N. Lipatov, Sov. J. Nucl. Phys. 28, 822 (1978).

[9] M. Derrick et al. [ZEUS Collaboration], Phys. Lett. B315, 481 (1993).

[10] T. Ahmed et al. [H1 Collaboration], Nucl. Phys. B429, 477 (1994).

[11] R. Bonino et al. [UA8 Collaboration], Phys. Lett. B211, 239 (1988).

[12] G. Ingelman and P. E. Schlein, Phys. Lett. B152, 256 (1985).

[13] A. Donnachie and P. V. Landshoff, Phys. Lett. B296, 227 (1992) hepph/9209205. 
[14] A. Donnachie and P. V. Landshoff, Nucl. Phys. B231, 189 (1984).

[15] K. Kang, J. R. Cudell, V. V. Ezhela, S. B. Lugovsky and N. P. Tkachenko, hep-ph/9812429.

[16] J. R. Forshaw and D. A. Ross, "Quantum Chromodynamics and the Pomeron," Cambridge University Press, $199 \%$.

[17] J. C. Collins, Phys. Rev. D57, 3051 (1998) hep-ph/9709499; erratum Phys. Rev. D61, 2000 (1998).

[18] A. Berera and D. E. Soper, Phys. Rev. D53, 6162 (1996) hep-ph/9509239.

[19] L. Trentadue and G. Veneziano, Phys. Lett. B323, 201 (1994).

[20] C. Adloff et al. [H1 Collaboration], Z. Phys. C76, 613 (1997) [hep-ex/9708016].

[21] J. Breitweg et al. [ZEUS Collaboration], Eur. Phys. J. C6, 43 (1999) hep$\mathrm{ex} / 9807010$.

[22] [ZEUS Collaboration], paper submitted to the European Conference on High Energy Physics, abstract 500, Tampere, 1999. (see also http://www-zeus.desy.de).

[23] [ZEUS Collaboration], paper submitted to the 29th International Conference on High Energy Physics, abstract 972, Vancouver, 1998. (see also http://wwwzeus.desy.de).

[24] H. Abramowicz and A. Levy, DESY 97-251, hep-ph/9712415.

[25] [H1 Collaboration], paper submitted to the 29th International Conference on High Energy Physics, abstract 571, Vancouver, 1998. (see also http://wwwh1.desy.de).

[26] [ZEUS Collaboration], paper submitted to the European Conference on High Energy Physics, abstract 524, Tampere, 1999. (see also http://www-zeus.desy.de).

[27] F. Hautmann, Z. Kunszt and D. E. Soper, hep-ph/9905218.

[28] [H1 Collaboration], paper submitted to the European Conference on High Energy Physics, abstract 157ae, Tampere, 1999. (see also http://www-h1.desy.de).

[29] [H1 Collaboration], paper submitted to the European Conference on High Energy Physics, abstract 157ag, Tampere, 1999. (see also http://www-h1.desy.de).

[30] [ZEUS Collaboration], paper submitted to the European Conference on High Energy Physics, abstract 527, Tampere, 1999. (see also http://www-zeus.desy.de). 
[31] H. Abramowicz and A. Caldwell, Rev. Mod. Phys. 71, 1275 (1999) hepex/9903037].

[32] A. Hebecker, hep-ph/9909504.

[33] J. Bartels, J. Ellis, H. Kowalski and M. Wuesthoff, Eur. Phys. J. C7, 443 (1999) hep-ph/9803497.

[34] A. Bialas, R. Peschanski and C. Royon, Phys. Rev. D57, 6899 (1998) hepph/9712216].

[35] W. Buchmuller, T. Gehrmann and A. Hebecker, Nucl. Phys. B537, 477 (1999) hep-ph/9808454.

[36] K. Golec-Biernat and M. Wuesthoff, Phys. Rev. D60, 114023 (1999) [hepph/9903358.

[37] E. Gotsman, E. Levin and U. Maor, Nucl. Phys. B493, 354 (1997) hepph/9606280.

[38] F. Hautmann, Z. Kunszt and D. E. Soper, Phys. Rev. Lett. 81, 3333 (1998) hep-ph/9806298.

[39] N. N. Nikolaev and B. G. Zakharov, hep-ph/9706343.

[40] M. G. Ryskin, J. Phys. G G22, 741 (1996).

[41] B. L. Ioffe, V. A. Khoze and L. N. Lipatov, "Hard Processes. Vol. 1: Phenomenology, Quark Parton Model," Amsterdam, Netherlands: North-Holland, 1984.

[42] W. Buchmuller, T. Gehrmann and A. Hebecker, hep-ph/9905321.

[43] B. Blaettel, G. Baym, L. L. Frankfurt and M. Strikman, Phys. Rev. Lett. 70, 896 (1993).

[44] L. Frankfurt and M. Strikman, hep-ph/9907221.

[45] A. H. Mueller, Eur. Phys. J. A1, 19 (1998) [hep-ph/9710531].

[46] L. Alvero, J. C. Collins, J. Terron and J. J. Whitmore, Phys. Rev. D59, 074022 (1999) hep-ph/9805268.

[47] F. Abe et al. [CDF Collaboration], Phys. Rev. Lett. 79, 2636 (1997).

[48] B. Abbott [D0 Collaboration], FERMILAB-PUB-99-375-E, submitted to Phys. Rev. Lett. hep-ex/9912061. 
[49] T. Affolder et al. [CDF Collaboration], FERMILAB-PUB-99-229-E, submitted to Phys. Rev. Lett.

[50] K. Borras [CDF Collaboration], FERMILAB-CONF-99-153-E, published in the proceedings of 7th International Workshop on Deep Inelastic Scattering and QCD (DIS 99), Zeuthen, Germany, 1999, Nucl. Phys. Proc. Sup. 79 (1999).

[51] F. Abe et al. [CDF Collaboration], Phys. Rev. Lett. 78, 2698 (1997) hepex/9703010].

[52] [CDF Collaboration], for recent results see http://www-cdf.fnal.gov/physics/new/qcd/QCD.html.

[53] M. G. Ryskin, Z. Phys. C57, 89 (1993).

[54] S. J. Brodsky, L. Frankfurt, J. F. Gunion, A. H. Mueller and M. Strikman, Phys. Rev. D50, 3134 (1994) hep-ph/9402283.

[55] T. H. Bauer, R. D. Spital, D. R. Yennie and F. M. Pipkin, Rev. Mod. Phys. 50, 261 (1978).

[56] [H1 Collaboration], paper submitted to the European Conference on High Energy Physics, abstract 157aj, Tampere, 1999. (see also http://www-zeus.desy.de).

[57] L. Frankfurt, W. Koepf and M. Strikman, Phys. Rev. D57, 512 (1998) [hepph/9702216.

[58] L. Frankfurt, W. Koepf and M. Strikman, Phys. Rev. D54, 3194 (1996) [hepph/9509311].

[59] C. Adloff et al. [H1 Collaboration], to appear in Eur. Phys. J. C hepex/9902019].

[60] J. Breitweg et al. [ZEUS Collaboration], Eur. Phys. J. C6, 603 (1999) hepex/9808020].

[61] J. Breitweg et al. [ZEUS Collaboration], Phys. Lett. B437, 432 (1998) [hepex/9807020].

[62] [H1 Collaboration], paper submitted to the 29th International Conference on High Energy Physics, abstract 574, Vancouver, 1998. (see also http://wwwh1.desy.de).

[63] L. L. Frankfurt, M. F. McDermott and M. Strikman, JHEP 9902, 002 (1999) hep-ph/9812316. 
[64] A. D. Martin, M. G. Ryskin and T. Teubner, Phys. Lett. B454, 339 (1999) hep-ph/9901420.

[65] A. V. Radyushkin, Phys. Rev. D56, 5524 (1997) hep-ph/9704207.

[66] X. Ji, J. Phys. G G24, 1181 (1998) hep-ph/9807358.

[67] C. Adloff et al. [H1 Collaboration], Eur. Phys. J. C10, 373 (1999) hepex/9903008.

[68] [ZEUS Collaboration], paper submitted to the 29th International Conference on High Energy Physics, abstract 793, Vancouver, 1998. (see also http://wwwzeus.desy.de).

[69] A. Levy, Phys. Lett. B424, 191 (1998) hep-ph/9712519.

[70] L. L. Frankfurt, A. Freund and M. Strikman, Phys. Rev. D58, 114001 (1998); erratum Phys. Rev. D59, 119901 (1999) hep-ph/9710356].

[71] [ZEUS Collaboration], paper submitted to the European Conference on High Energy Physics, Tampere, 1999. (see also http://www-zeus.desy.de).

[72] T. Ishikawa et al., GRAPE-Compton Monte Carlo generator, KEK Report 92-19 (1993).

[73] P. Saull, GenDVCS Monte Carlo generator, available from http://wwwzeus.desy.de/ saull/public/dvcs.htm, 1999.

[74] F. Abe et al. [CDF Collaboration], Phys. Rev. Lett. 81, 5278 (1998).

[75] B. Abbott et al. [D0 Collaboration], Phys. Lett. B440, 189 (1998) [hepex/9809016].

[76] B. Cox [H1 Collaboration], published in the proceedings of 7th International Workshop on Deep Inelastic Scattering and QCD (DIS 99), Zeuthen, Germany, 1999, Nucl. Phys. Proc. Sup. 79 (1999).

[77] [CDF Collaboration], for recent results see http://www-cdf.fnal.gov/physics/new/qcd/QCD.html.

\section{Discussion}

Harry Lipkin (Weizmann Institute): I would like to point out that the Donnachie and Landshoff fit to the total cross section, which was accepted by the Particle 
Data Group in 1966, was thrown out by them in 1998 with a different value of the pomeron intercept. I have fit the same data some time ago with a still different value. The point is that there are still not enough data on total cross sections to tell us whether there is a universal pomeron with a universal intercept that is coupled to meson-baryon and baryon-baryon total cross sections. What are needed are new data at higher energies. The recent data on hyperon-nucleus cross sections from SELEX actually support my model, but I don't believe that either. I think that what is happening is that there is a non-leading contribution which confuses the issue and is not understood. To resolve this, we just have to get more data at higher energies.

Answer: There is no denying that a well "measured" soft Pomeron would help to establish when the departure from universality becomes effective. However, it is difficult to imagine that it would alter in any way the fact that the $W$ dependence of the total $\gamma^{\star} p$ cross section changes with $Q^{2}$ and follows the laws of QCD evolution. I think that even the diffractive data, imprecise as they are, cannot be accommodated by a universal trajectory. It may well be that the idealized Pomeron of Gribov is just a mathematical construction, while the pomeron we try to investigate is just another name for the nature of strong interactions. The latter, by virtue of QCD and the interplay of soft and hard physics, remain versatile. 